\title{
Neoadjuvant Therapy of Gastric Cancer: A Decisive Step Forward
}

\author{
Markus Menges ${ }^{a} \quad$ Thomas Hoehler $^{b}$ \\ ${ }^{a}$ Department of Internal Medicine 2, Diakonie-Hospital, Schwäbisch Hall, and \\ ${ }^{b}$ Department of Internal Medicine 1, Prosper Hospital, Recklinghausen, Germany
}

\section{Key Words}

Gastric cancer - Cancer of the esophagogastric junction - Neoadjuvant chemotherapy . Neoadjuvant radiochemotherapy · Improvement of prognosis

\begin{abstract}
Background: Although its incidence has been steadily decreasing in Western countries, gastric cancer remains a leading cause of cancer deaths worldwide. The detection rate of earlystage cancers is improving; nevertheless, the majority of cases is still diagnosed at later stages with a poor prognosis. Furthermore, the results that can be achieved with surgery have reached a plateau of effectiveness.

Summary: Neoadjuvant chemotherapy was successfully introduced first in patients with noncuratively resectable disease. In the last decade, neoadjuvant chemotherapy has also been established in potentially curatively resectable cases and has become the state-of-the-art treatment. Esophagogastric junction (EGJ) tumors are not optimally treated with chemotherapy alone, and combined radiochemotherapy (RCT) seems to yield superior outcomes.

Key Message: The use of neoadjuvant therapy has been successfully established in patients with curatively resectable disease. Neoadjuvant chemotherapy is now a cornerstone in the treatment of gastric cancer and cancer of the EGJ, although further work is needed in order to define the optimal combination regimen.

Practical Implications: Neoadjuvant chemotherapy is currently the gold standard for the treatment of gastric cancer and cancer of the EGJ. Several independent studies have shown the benefits of using combination regimens that included cisplatin and 5-fluorouracil, though recently the use of the EOX (epirubicin, oxaliplatin and capecitabine) regimen has been widely accepted in this setting. Tumors of the EGJ benefit from neoadjuvant treatment with combined RCT. It should be noted that the optimal neoadjuvant regimen in EGJ tumors has not yet been defined, and the survival advantage of neoadjuvant RCT over neoadjuvant chemotherapy remains to be established in this patient population.




\section{Introduction}

Although its incidence has been steadily decreasing in Western countries for several decades, gastric cancer remains to be an important health issue and a leading cause of cancer deaths worldwide [1]. The current yearly incidence varies from 5 to 10 per 100,000 in Western Europe and the USA to over 70 in some Far East countries [2, 3]. Whereas the frequency of the 'classic' gastric cancer (i.e. Lauren's intestinal type with its predominant location in the corpus und antrum) is decreasing, there has been a shift to proximal cancer types involving the cardia and the distal esophagus, which are histologically of the diffuse type and usually present at a more advanced stage with a significantly worse prognosis [4-6].

Except for early gastric cancers (T1 according to the TNM classification), which may be curatively treated by endoscopic resection alone, radical surgery still offers the only chance of cure for all other cases, which still represent $80-90 \%$ in countries with a lower overall incidence. In countries with high gastric cancer incidence like Japan and South Korea, the implementation of screening programs increased the recognition rate of early cancers to over $40 \%$ in specialized centers. However, efforts to increase the rate of early cancer detection in other parts of the world have not yet been satisfactory. About two thirds of gastric cancer patients are diagnosed at an advanced clinical stage of disease, mainly stage III and IV (Union for International Cancer Control, UICC).

Thus, curative surgical resection is difficult, and in many cases unreachable. In the large multicentric German Gastric Carcinoma Study enrolling almost 2,000 patients, the R0 resection rate in UICC stage III patients was only 41.1\% [7]. Even under optimal surgical conditions with extended lymphadenectomy performed by highly skilled surgeons in the large European surgical trials, which compared D1 versus D2 compartment lymphadenectomy, about $40 \%$ of the patients were not curatively resectable [8, 9]. Moreover, radical D2 lymphadenectomy did not result in a better long-term prognosis [10], suggesting that surgery has reached a plateau of effectiveness.

\section{Neoadjuvant Therapy in Patients Not Curatively Resectable due to Locally Advanced Cancer}

Neoadjuvant therapy aims at 'downstaging' the tumor and eliminating the spread of tumor cells or lymph node metastases. This concept was first tested in patients with advanced gastric cancer, for whom curative resection was considered impossible. Studies including patients who were staged by a surgeon at laparotomy and then underwent chemotherapy when considered to be 'irresectable' are of great interest.

Understandably, these studies enrolled only small numbers of patients, but demonstrated a significant benefit for those patients in that about $40 \%$ of them were R0 resected after 2 or 3 cycles of systemic chemotherapy [11-16]. The Italian group could confirm these results in a larger trial enrolling 82 patients, who were considered not curatively resectable according to laparotomy in $63 \%$ and to CT scans in the remaining third. After polychemotherapy $45 \%$ of the patients achieved R0 resection. This, in turn, resulted in a significantly better prognosis: 25 of 37 resected patients were alive after 2 years and 24 of them were disease-free. The median and 4-year survival of the whole group was 17 months and 31\%, respectively [17]. Due to ethical reasons phase III studies could not be performed in this setting. 


\section{Neoadjuvant Chemotherapy in Potentially (Curatively) Resectable Patients}

About two decades later the neoadjuvant treatment principle was transferred to patients who were considered to be curatively resectable. Cunningham et al. [18] performed a randomized study on 503 patients suffering from upper gastrointestinal cancer, mainly gastric cancer $(74 \%)$. The study protocol consisted of three pre- and three postoperative cycles of epirubicin, cisplatin and 5-fluorouracil (5-FU) for the combined therapy group versus a control group without chemotherapy. The resection in the combined therapy group was performed after three cycles of chemotherapy regardless of patient response. The results were impressive: the rate of R0 resections was $79 \%$ in the combined group versus $69 \%$ in the control group, and the overall 5-year survival rate was 36 vs. $23 \%(\mathrm{p}=0.009)$. In fact, only $40 \%$ of the patients in the combined arm received the intended postoperative chemotherapy, mainly due to intolerance in the postoperative phase. Therefore it can be postulated that the preoperative treatment is decisive for the beneficial effects.

The next question was whether similar results could be achieved with a more simple chemotherapy regimen. Ychou et al. [19] published a randomized study on 224 patients with upper gastrointestinal cancer, of whom the majority (64\%) had adenocarcinomas of the esophagogastric junction (EGJ) (associated with a poor prognosis). Two or three cycles of a cisplatin/5-FU regimen followed by resection and (in responders) postoperatively the same regimen led to a significant increase in 5-year survival in the combined treatment arm (38 vs. $24 \%, \mathrm{p}=0.02$ ).

Not all results of studies on neoadjuvant chemotherapy in gastric cancer were consistent: The European Organisation for Research and Treatment of Cancer conducted another randomized trial to compare neoadjuvant chemotherapy plus surgery with surgery alone in patients with advanced cancer of the stomach and the cardia [20]. The trial was stopped for poor accrual when 144 patients were enrolled. Although the $\mathrm{R} 0$ resection rate in the combined therapy group was significantly higher ( 81.9 vs. $66.7 \%, p=0.036)$, a survival benefit could not be shown. The authors explain this with a low statistical power, a high rate of proximal (EGJ) cancers of $52 \%$ and a better outcome than expected in the surgery alone group due to extended lymph node dissection outside the perigastric area.

Meanwhile we learned from the large REAL studies in the palliative setting that 5-FU may be substituted by the oral fluoropyrimidine capecitabine and cisplatin by the less toxic oxaliplatin without any loss of efficacy. This substitution simplifies perioperative chemotherapy, and EOX (epirubicin, oxaliplatin and capecitabine) has currently been established widely as a standard regimen for this purpose.

\section{Neoadjuvant Radiochemotherapy in Potentially (Curatively) Resectable Patients with Esophagogastric Junction Tumors}

The incidence of adenocarcinoma of the EGJ has been increasing dramatically over the last decades. Treatment outcomes with surgery alone have shown disappointing results. There are only very few trials focusing exclusively on patients with adenocarcinoma of the EGJ. Most trials incorporated patients with squamous cell carcinoma, which has a different biology. Adenocarcinoma patients constituted a subgroup of the trial population.

In the Dutch CROSS trial, $75 \%$ of 363 patients had potentially resectable adenocarcinoma of the distal esophagus and the EGJ [21]. Patients receiving neoadjuvant weekly paclitaxel plus carboplatin with concurrent radiotherapy (41.4 Gy over 5 weeks) had pathologic complete responses in 29\% and a significantly higher resection rate (R0 resections 92 vs. $65 \%$ ) than patients in the surgery alone arm. The 3-year survival rate was $58 \%$ in the neoadjuvant radio- 
chemotherapy (RCT) arm versus $44 \%$ in the controls. These results confirmed data from a smaller Irish trial comprising 113 patients with esophageal adenocarcinoma [22]. Neoadjuvant RCT consisting of 5-FU and cisplatin with radiotherapy of $40 \mathrm{~Gy}$ in this trial resulted in pathologic complete response in $25 \%$ of patients. However, this trial has been criticized because of its low survival rates. The 3 -year survival rate was $32 \%$ in the neoadjuvant arm compared to only $6 \%$ in the surgery alone group. A survival advantage of preoperative RCT was also shown in the CALGB 9781 trial [23]. A fourth small randomized trial with 43 patients did not demonstrate a survival advantage but was probably underpowered [24].

The optimal neoadjuvant regimen has not yet been established since neoadjuvant RCT trials used different chemotherapy and radiation schedules $[25,26]$. Only two trials compared neoadjuvant RCT with chemotherapy alone. In the German POET study neoadjuvant RCT with cisplatin, 5-FU, leucovorin and 30 Gy radiotherapy was compared to chemotherapy with a similar regimen, both arms followed by surgery [25]. Complete pathologic response was achieved in $16 \%$ in the RCT and in $2 \%$ in the chemotherapy arm. The 3-year survival rate was not significantly improved with $47 \%$ (RCT) vs. $28 \%$ in the chemotherapy arm. This trial was ended prematurely due to poor accrual with only 126 of 354 planned patients. An Australian phase II trial with 75 patients with adenocarcinoma of the distal esophagus and EGJ also showed an improved histopathological response rate and a higher R0 resection rate with neoadjuvant RCT compared to chemotherapy alone [26]. However, neoadjuvant RCT did not improve overall survival.

The most recent meta-analysis of neoadjuvant RCT in adenocarcinomas of the distal esophagus and the esophageal junction showed a survival benefit of $8.7 \%$ at 2 years compared to surgery alone [27]. Compared to neoadjuvant chemotherapy RCT shows improved locoregional control, but a clear survival advantage of neoadjuvant RCT over neoadjuvant chemotherapy has yet to be established.

\section{Future Aspects}

The next logical step aims at a better (earlier) identification of responders and nonresponders to preoperative chemotherapy. All in all, about $70 \%$ of gastric cancer patients respond to the various currently applied chemotherapy protocols. There have been attempts to select the non-responders by evaluation of their biologic tumor activity revealed by positron emission tomography shortly after initiation of chemotherapy. In patients who did not show a significant decrease of their biologic tumor activity 2 weeks after initiation of chemotherapy, this could be discontinued without compromising the prognosis [28]. So far, this has not been confirmed in large multicenter trials, which is partly due to a restricted access to positron emission tomography.

Another point of interest is the question which role postoperative chemotherapy plays in the whole setting. Undoubtedly, postoperative chemotherapy, especially when gastrectomy was performed, is less well tolerated than prior to operation. In both large randomized studies the rate of fully applied postoperative chemotherapy cycles was less than $50 \%$. We know from the large number of studies addressing adjuvant chemotherapy that the benefit of the patients is very small if there is one at all [29-32]. To evaluate the role of postoperative therapy, randomized studies are needed which compare perioperative (pre- and postoperative therapy) with pure neoadjuvant treatment.

In conclusion, as long as the majority of gastric cancer patients is diagnosed at an advanced stage of disease, neoadjuvant treatment will be the standard of care and has already improved the prognosis of these patients more than any other development in the treatment of gastric cancer during the last 20 years. 


\section{References}

1 Varadhachary G, Ajani JA: Gastric cancer. Clin Adv Hematol Oncol 2005;3:118-124.

$\longrightarrow 2$ Crew KD, Neugut AI: Epidemiology of gastric cancer. World J Gastroenterol 2006;12:354-362.

-3 Shah MA, Kelsen DP: Gastric cancer: a primer on the epidemiology and biology of the disease and an overview of the medical management of advanced disease. J Natl Compr Canc Netw 2010;8:437-447.

4 Sakaguchi T, Watanabe A, Sawada H, Yamada Y, Tatsumi M, Fujimoto H, Emoto K, Nakano H: Characteristics and clinical outcome of proximal-third gastric cancer. J Am Coll Surg 1998;187:352-357.

-5 Hochwald SN, Kim S, Klimstra DS, Brennan MF, Karpeh MS: Analysis of 154 actual 5-year survivors of gastric cancer. J Gastrointest Surg 2000;4:520-525.

6 Kim DY, Joo JK, Park YK, Ryu SY, Kim YJ, Kim SK: Predictors of long-term survival in node-positive gastric carcinoma patients with curative resection. Langenbecks Arch Surg 2007;392:131-134.

7 Roder JD, Böttcher K, Siewert JR, Busch R, Hermanek P, Meyer HJ: Prognostic factors in gastric carcinoma. Results of the German Gastric Carcinoma Study 1992. Cancer 1993;72:2089-2097.

-8 Bonenkamp JJ, Songun I, Hermans J, Sasako M, Welvaart K, Plukker JT, van Elk P, Obertop H, Gouma DJ, Taat CW: Randomised comparison of morbidity after D1 and D2 dissection for gastric cancer in 996 Dutch patients. Lancet 1995;345:745-748.

-9 Cuschieri A, Weeden S, Fielding J, Bancewicz J, Craven J, Joypaul V, Sydes M, Fayers P: Patient survival after D1 and D2 resections for gastric cancer: long-term results of the MRC randomized surgical trial. Surgical Co-operative Group. Br J Cancer 1999;79:1522-1530.

10 Hartgrink HH, van de Velde CJ, Putter H, Bonenkamp JJ, Klein Kranenbarg E, Songun I, Welvaart K, van Krieken JH, Meijer S, Plukker JT, van Elk PJ, Obertop H, Gouma DJ, van Lanschot JJ, Taat CW, de Graaf PW, von Meyenfeldt MF, Tilanus H, Sasako M: Extended lymph node dissection for gastric cancer: Who may benefit? Final results of the randomized Dutch gastric cancer group trial. J Clin Oncol 2004;22:2069-2077.

-11 Wilke H, Preusser P, Fink U, Gunzer U, Meyer HJ, Meyer J, Siewert JR, Achterrath W, Lenaz L, Knipp H: Preoperative chemotherapy in locally advanced and nonresectable gastric cancer: a phase II study with etoposide, doxorubicin, and cisplatin. J Clin Oncol 1989;7:1318-1326.

-12 Plukker JT, Mulder NH, Sleijfer DT, Grond J, Verschueren RC: Chemotherapy and surgery for locally advanced cancer of the cardia and fundus: phase II study with methotrexate and 5-fluorouracil. Br J Surg 1991;78:955958.

13 Lerner A, Gonin R, Steele GD Jr, Mayer RJ: Etoposide, doxorubicin, and cisplatin chemotherapy for advanced gastric adenocarcinoma: results of a phase II trial. J Clin Oncol 1992;10:536-540.

14 Cascinu S, Labianca R, Graziano F, Pancera G, Barni S, Frontini L, Luporini G, Cellerino R, Catalano G: Intensive weekly chemotherapy for locally advanced gastric cancer using 5-fluorouracil, cisplatin, epidoxorubicin, 6S-leucovorin, glutathione and filgrastim: a report from the Italian Group for the Study of Digestive Tract Cancer (GISCAD). Br J Cancer 1998;78:390-393.

15 Gallardo-Rincon D, Onate-Ocana LF, Calderillo-Ruiz G: Neoadjuvant chemotherapy with P-ELF (cisplatin, etoposide, leucovorin, 5-fluorouracil) followed by radical resection in patients with initially unresectable gastric adenocarcinoma: a phase II study. Ann Surg Oncol 2000;7:45-50.

-16 Menges M, Schmidt C, Lindemann W, Ridwelski K, Pueschel W, Jüngling B, Feifel G, Schilling M, Stallmach A, Zeitz M: Low toxic neoadjuvant cisplatin, 5-fluorouracil and folinic acid in locally advanced gastric cancer yields high R-0 resection rate. J Cancer Res Clin Oncol 2003;129:423-429.

-17 Cascinu S, Scartozzi M, Labianca R, Catalano V, Silva RR, Barni S, Zaniboni A, D’Angelo A, Salvagni S, Martignoni G, Beretta GD, Graziano F, Berardi R, Franciosi V; Italian Group for the Study of Digestive Tract Cancer (GISCAD): High curative resection rate with weekly cisplatin, 5-fluorouracil, epidoxorubicin, 6S-leucovorin, glutathione, and filgastrim in patients with locally advanced, unresectable gastric cancer: a report from the Italian Group for the Study of Digestive Tract Cancer (GISCAD). Br J Cancer 2004;90:1521-1525.

-18 Cunningham D, Allum WH, Stenning SP, Thompson JN, Van de Velde CJ, Nicolson M, Scarffe JH, Lofts FJ, Falk SJ, Iveson TJ, Smith DB, Langley RE, Verma M, Weeden S, Chua YJ: Perioperative chemotherapy versus surgery alone for resectable gastroesophageal cancer. N Engl J Med 2006;355:11-20.

-19 Ychou M, Boige V, Pignon JP, Conroy T, Bouché O, Lebreton G, Ducourtieux M, Bedenne L, Fabre JM, SaintAubert B, Genève J, Lasser P, Rougier P: Perioperative chemotherapy compared with surgery alone for resectable gastroesophageal adenocarcinoma: an FNCLCC and FFCD multicenter phase III trial. J Clin Oncol 2011;29:1715-1721.

20 Schuhmacher C, Gretschel S, Lordick F, Reichardt P, Hohenberger W, Eisenberger CF, Haag C, Mauer ME, Hasan B, Welch J, Ott K, Hoelscher A, Schneider PM, Bechstein W, Wilke H, Lutz MP, Nordlinger B, Van Cutsem E, Siewert JR, Schlag PM: Neoadjuvant chemotherapy compared with surgery alone for locally advanced cancer of the stomach and cardia: European Organisation for Research and Treatment of Cancer randomized trial 40954. J Clin Oncol 2010;28:5210-5218.

21 van Hagen P, Hulshof MC, van Lanschot JJ, Steyerberg EW, van Berge Henegouwen MI, Wijnhoven BP, Richel DJ, Nieuwenhuijzen GA, Hospers GA, Bonenkamp JJ, Cuesta MA, Blaisse RJ, Busch OR, ten Kate FJ, Creemers GJ, Punt CJ, Plukker JT, Verheul HM, Spillenaar Bilgen EJ, van Dekken H, van der Sangen MJ, Rozema T, Biermann K, Beukema JC, Piet AH, van Rij CM, Reinders JG, Tilanus HW, van der Gaast A: Preoperative chemoradiotherapy for esophageal or junctional cancer. N Engl J Med 2012;366:2074-2084. 
22 Walsh TN, Noonan N, Hollywood D, Kelly A, Keeling N, Hennessy TP: A comparison of multimodal therapy and surgery for esophageal adenocarcinoma. N Engl J Med 1996;335:462-467.

-23 Tepper J, Krasna MJ, Niedzwiecki D, Hollis D, Reed CE, Goldberg R, Kiel K, Willett C, Sugarbaker D, Mayer R: Phase III trial of trimodality therapy with cisplatin, fluorouracil, radiotherapy, and surgery compared with surgery alone for esophageal cancer: CALGB 9781. J Clin Oncol 2008;26:1086-1092.

-24 Urba SG, Orringer MB, Turrisi A, Iannettoni M, Forastiere A, Strawderman M: Randomized trial of preoperative chemoradiation versus surgery alone in patients with locoregional esophageal carcinoma. J Clin Oncol 2001; 19:305-313.

-25 Stahl M, Walz MK, Stuschke M, Lehmann N, Meyer HJ, Riera-Knorrenschild J, Langer P, Engenhart-Cabillic R, Bitzer M, Königsrainer A, Budach W, Wilke H: Phase III comparison of preoperative chemotherapy compared with chemoradiotherapy in patients with locally advanced adenocarcinoma of the esophagogastric junction. J Clin Oncol 2009;27:851-856.

-26 Burmeister BH, Thomas JM, Burmeister EA, Walpole ET, Harvey JA, Thomson DB, Barbour AP, Gotley DC, Smithers BM: Is concurrent radiation therapy required in patients receiving preoperative chemotherapy for adenocarcinoma of the oesophagus? A randomised phase II trial. Eur J Cancer 2011;47:354-360.

-27 Sjoquist KM, Burmeister BH, Smithers BM, Zalcberg JR, Simes RJ, Barbour A, Gebski V; Australasian GastroIntestinal Trials Group: Survival after neoadjuvant chemotherapy or chemoradiotherapy for resectable oesophageal carcinoma: an updated meta-analysis. Lancet Oncol 2011;12:681-692.

28 Lordick F, Ott K, Krause BJ, Weber WA, Becker K, Stein HJ, Lorenzen S, Schuster T, Wieder H, Herrmann K, Bredenkamp R, Höfler H, Fink U, Peschel C, Schwaiger M, Siewert JR: PET to assess early metabolic response and to guide treatment of adenocarcinoma of the oesophagogastric junction: the MUNICON phase II trial. Lancet Oncol 2007;8:797-805.

29 Hermans J, Bonenkamp JJ, Boon MC, Bunt AM, Ohyama S, Sasako M, Van de Velde CJ: Adjuvant therapy after curative resection for gastric cancer: meta-analysis of randomized trials. J Clin Oncol 1993;12:879-880.

30 Earle CC, Maroun JA: Adjuvant chemotherapy after curative resection for gastric cancer in non-Asian patients: revisiting a meta-analysis of randomized trials. Eur J Cancer 1999;35:1059-1064.

-31 Mari E, Floriani I, Tinazzi A, Buda A, Belfiglio M, Valentini M, Cascinu S, Barni S, Labianca R, Torri V: Efficacy of adjuvant chemotherapy after curative resection for gastric cancer: a meta-analysis of published randomised trials. A study of the GISCAD. Ann Oncol 2000;11:837-843.

-32 Janunger KG, Hafstroem L, Glimelius B: Chemotherapy in gastric cancer: a review and updated meta-analysis. Eur J Surg 2002;168:597-608. 